\title{
Design of the Hardware Scheme of the UAV Electronic Governor System $\mathrm{Li} \mathrm{Li}^{\mathrm{a}}$, Xia Liu and Yang Zhao ${ }^{\mathrm{*}}$ \\ Department of Mechanical and Electrical Engineering, Guangdong University of Science \& Technology, Dongguan, China \\ a65799279@qq.com, b294057303@qq.com, czhaoyangmtap@163.com
}

Keywords: Electronic governor system; Hardware scheme; Unmanned aerial vehicle; Power module

\begin{abstract}
The traditional aero model DC(direct current) motor is commutated mechanically by brush and commutator, however, there is mechanical friction between the brush and commutator, which will cause noise, sparks, electromagnetic interference and many other problems. Because of the disadvantages of high manufacturing cost, short service life and difficult maintenance, the use of brush motors is restricted in many situations. The DC brushless motor is the rotation of the magnetic pole, while the armature is still. The electronic inverter is controlled by the magnetic pole position signal to supply the power to the armature. Using the electronic inverter to replace the mechanical commutator has a very obvious advantage. This paper designs a UAV(unmanned aerial vehicle) brushless motor speed controller based on SCM(single chip microcomputer), mainly in order to meet the light weight, high control precision, good stability and other special requirements of the UAV brushless motor controller. The results show that the design uses fewer components to realize the start, stop, forward and reverse rotation, acceleration and deceleration, emergency stop and other functions.
\end{abstract}

\section{Introduction}

In the aeromodelling field, traditional brush motor hasn't already meet the aeromodelling system's special requirements of the high precision, low power consumption, stability, light weight[1-2]. Brushless motor with small size, light weight, low cost, no commutation spark, no carbon brush wear, long service life, etc [3-5]. It will replace the traditional brush motor has become inevitable[6-8]. In addition, as the global price of brushless motor speed controller has greatly decreased, also make the brushless motor has been widely applied.

At present, electronic products are experiencing the conversion from analog to digital, and the development of power electronics technology, computer technology and control theory makes the motor speed control technology developed rapidly[9]. The application of new power electronic devices and digital integrated circuit with high performance and advanced control theory makes the function of the control unit more perfect[10]. The number and controller required less control device volume is shrinking, which improve the reliability of the controller and the cost is lower.

\section{The Overall Hardware Scheme of the UAV Electronic Governor System}

The hardware of electronic governor system of UAV is mainly composed of STC89C52RC main controller, a brushless DC motor, 7407I/O driver circuit, TLP521 optocoupler isolation circuit and three-phase full bridge circuit, Holzer sensor phase detection circuit, key matrix circuit and LED digital tube display circuit.

The controller STC89C52RC is used for the hardware design of UAV brushless motor speed controller based on MCU and the motor uses a 12-36V brushless motor. The drive circuit of power module comprises a $12 \mathrm{~V}$ power supply and $5 \mathrm{~V}$ control module. There are two isolation circuits in electronic governor system: the isolation circuit between the three-phase full bridge circuit and the 7407 microcontroller I/O port and the isolation circuit between the driving circuit of Holzer sensor phase detection circuit and the MCU P3 port. The speed display circuit of brushless motor is made up of 
74LS138 and 747LS48. The three-phase full bridge is composed of IRF4905 FET with upper arm P channel, and STP60NF06 field effect transistor with lower arm N channel.

The Minimum System Circuit of the Electronic Governor. STC89C52RC is a low power, high performance CMOS8 bit microcontroller produced by STC, and it has a programmable Flash memory of $8 \mathrm{~K}$. The minimum system of the microcontroller of the electronic governor is shown in Figure 1.

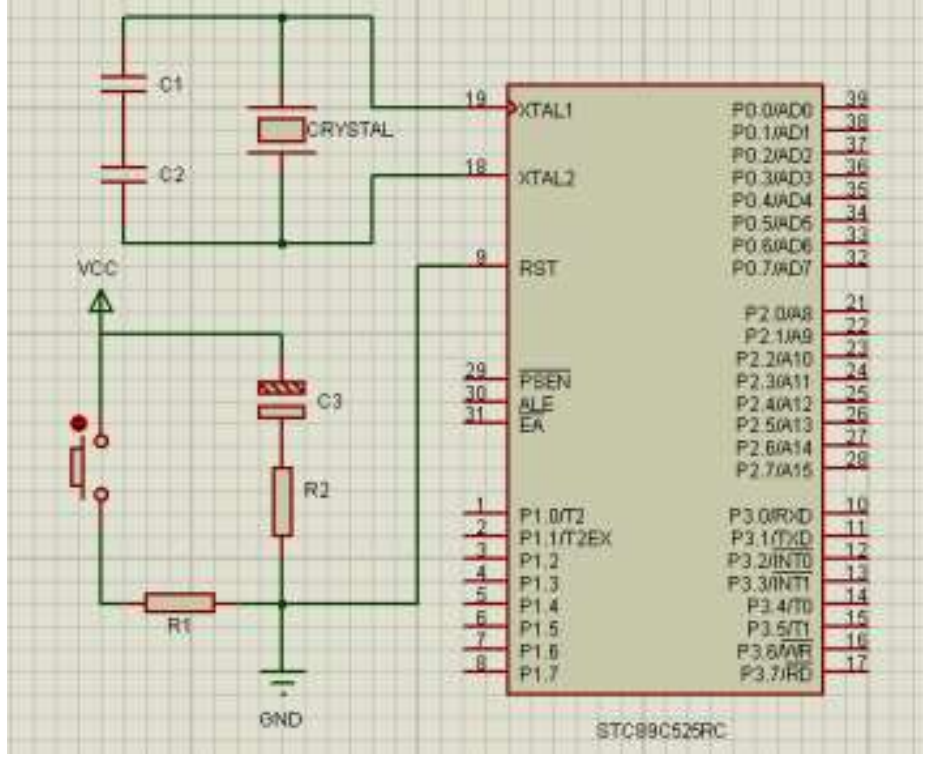

Figure 1. Minimum system circuit diagram of electronic governor

Power Module Selection. The design uses single power supply, and the control module power is built by AMS1117-5.0, which is composed of $12 \mathrm{~V}$ input and $5 \mathrm{~V}$ output voltage regulator module. The driving module power supply adopts ONK-0122000 power adapter with AC220V input and DC12V/3A output. The two part of the power supply is stable and reliable, which solves the problem of the power supply of the system. The $12 \mathrm{~V}$ input to $5 \mathrm{~V}$ output regulator module of the AMS1117-5.0 is shown in Figure 2.

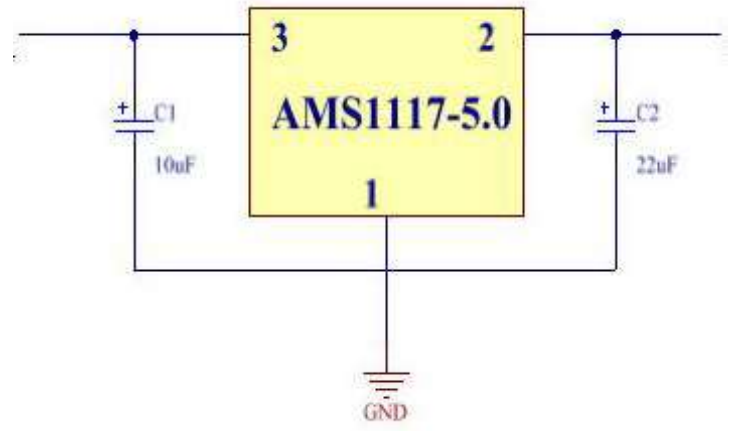

Figure 2. AMS1117-5.0 power supply for 5V output

Introduction of I/O Port Drive Circuit. The output signal of I/O port of STC series microcontroller is weak. In order to enhance the driving capability of I/O port of the microcontroller, the driver chip 7407 is used in the design scheme. Chip 7407 is the six high voltage output buffer / driver, which mainly plays the role of cushioning. It is the ability to buffer the microcontroller. It uses 7407 to amplify the output current of the singlechip, so that the driving ability is enhanced. The 7407 drive circuit is as shown in Figure 3. 


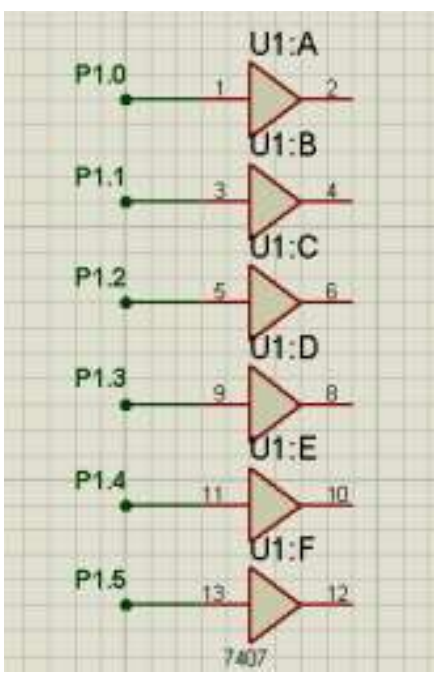

Figure 3. The I/O port drive circuit

Three Phase Full Bridge Circuit. In this design, the upper bridge arm uses the $\mathrm{P}$ channel low level conduction IRF4905 and the lower bridge arm uses the N channel high level conduction STP60NF06. The specific circuit diagram of the three phase full bridge circuit is shown in Figure 4.

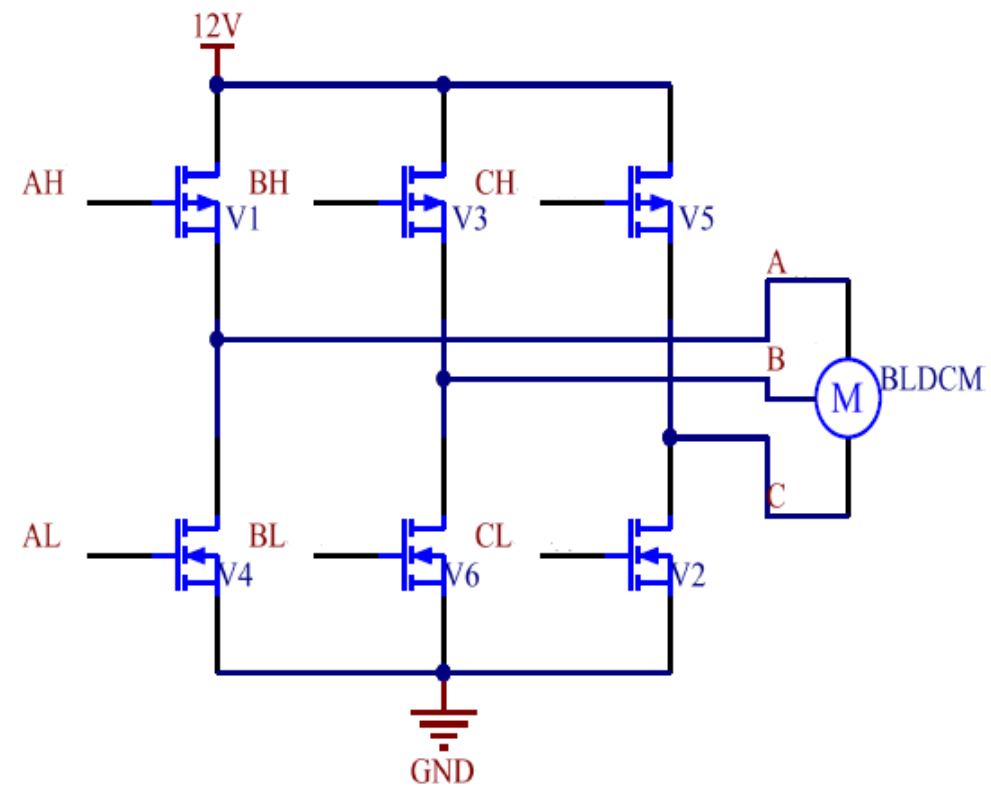

Figure 4. Three phase full bridge circuit

Optocoupler Isolation Circuit. The design of optocoupler isolation circuit is to eliminate the interference of external signals to SCM. In this design, the optocoupler isolation circuit includes two parts: the 7407 driving circuit and the three-phase full bridge between the Holzer signal and the microcontroller. The optocoupler isolation circuit is composed of integrated chip TLP521-1, TLP521-2 and TLP521-4. The hardware optocoupler circuit is as shown in Figure 4. 


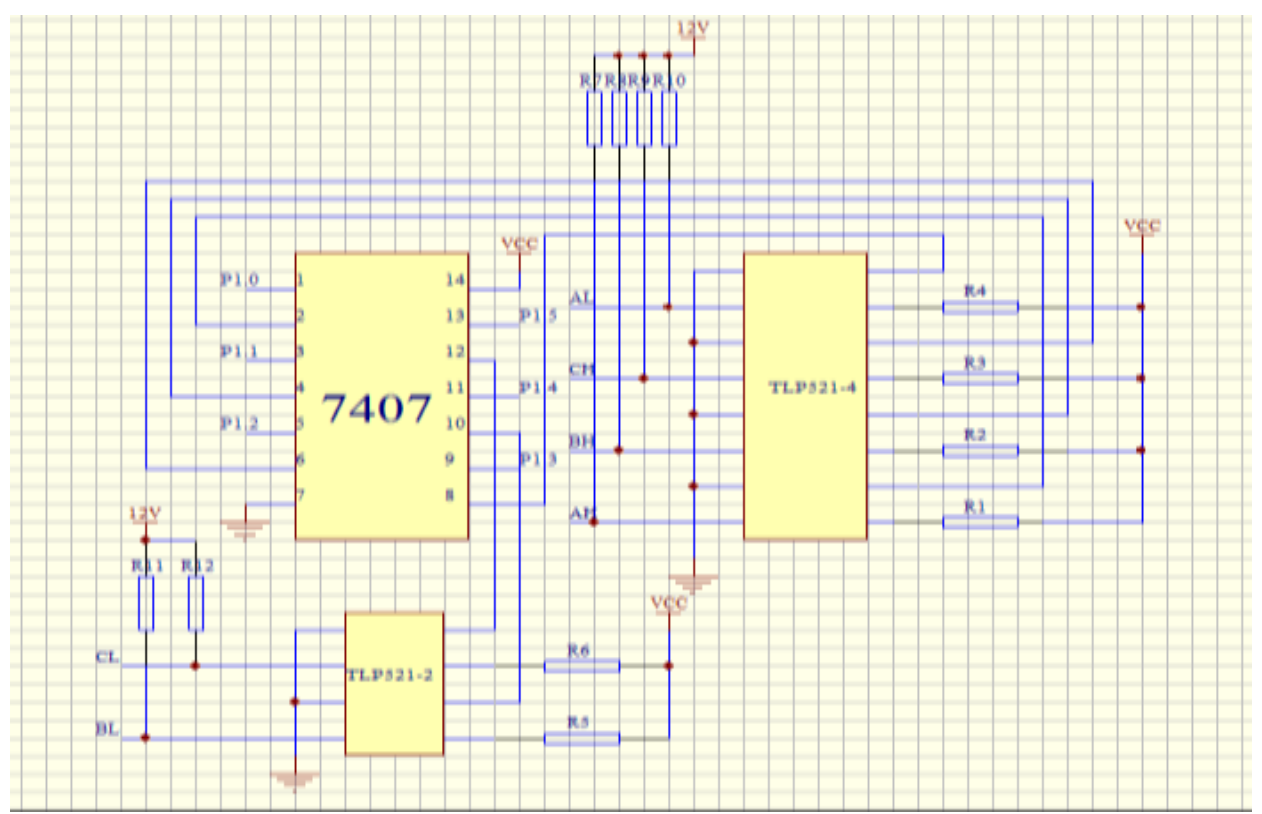

Figure 4. The hardware optocoupler circuit

\section{The Motor Speed Formula}

Basic equations of DC motor is

$$
\begin{aligned}
& U=E+R_{a} I_{a} \\
& E=C_{E} \Phi n \\
& T_{e}=T_{L}=T_{2}+T_{0} \\
& T_{e}=C_{T} \Phi I_{a} \\
& P_{e}=P_{2}+P_{0}
\end{aligned}
$$

The formula of motor speed can be derived from the above formula,

$$
n=\frac{U}{C_{E} \Phi}-\frac{R_{a}}{C_{E} \Phi} I_{a}
$$

\section{Summary}

This design is based on MCU's aeromodelling brushless motor speed controller. It main solves the problem of model aircraft system's stability and accuracy.The system uses STC89C52RC as core controller, motor adopts inductive brushless motor, 7407 driver chip enhances the driver capacity of MCU I/O port, controlling the on-off state of the six Power Mosfets to realize the commutation of three-phase brushless motor, brushless motor by hall sensor to realize the rotor position's detection, motor speed through the LED digital tube to display, software programming in assembly language. In addition, in the design of the hardware also used TLP521 optical coupling isolation circuit to anti-interference, over-current protection circuit to protect the motor. This design also needs to improve in some aspects, it provides a space for system's upgrading and optimization.

\section{Acknowledgements}

This work is supported by Project of Dongguan Social Science and Technology Development. 


\section{References}

[1] SH Weon, GY Kim, JH Cha, KH Park, And HI Choi. A Mapping Method For 3D Satellite And Sensor Images Using A Road Extraction Algorithm For Occlusion Processing Of Virtual Targets, International Journal Of Computer Applications In Technology, 46 (2013), 45-53.

[2] Juan Ren. Clustering Method And Relevant Application Of Multi-Index Panel Data, Statistics And Decision, 352(2012) , 92-95.

[3] Vitartas, Peter, S. Kinnear, And K. Charters. Supporting Innovation In Regional Australia - Perceptions From Regional And Urban Innovators, Journal Of Economic \& Social Policy, 15(2013), 76-85.

[4] Brekke, Asgeir. Physics Of The Upper Polar Atmosphere, Atmospheric Environment, 32(2012), 262-275.

[5] NS Abeysingha, S Man, VK Sehgal, M Khanna, And H Pathak. Analysis Of Rainfall And Temperature Trends In Gomti River Basin, Sustainability, 14(2014), 56-66.

[6] Tsay, And Ruey S. Multivariate Time Series Analysis With R And Financial Applications, Wiley, 3 (2013), $1-40$.

[7] Sapucci, And Luiz F. Evaluation Of Modeling Water-Vapor-Weighted Mean Tropospheric Temperature For GNSS-Integrated Water Vapor Estimates In Brazil, Journal Of Applied Meteorology \& Climatology, 53(2013), 715-730.

[8] Karstensen, Jonas, G. P. Peters, And R. M. Andrew. Allocation Of Global Temperature Change To Consumers, Climatic Change, 129(2015), 43-55.

[9] Bonzo D C And Hermosilla A Y. Clustering Panel Data Via Perturbed Adaptive Simulated Annealing And Genetic Algorithms, Advances In Complex Systems, 26 (2012),339-360.

[10] Chenglin Tan , Yunfeng Zheng, And Zhanghua. Trend And Characteristic Analysis For Coordinated Development Of Regional Economy In China, Economic Geography, 33 (2013),Pp. 9-14. 